\title{
Análisis factorial aplicado en la evaluación de competencias emprendedoras
}

\section{Applied factor analysis in the evaluation of entrepreneurial competencies}

\author{
MARTINEZ-MONTAÑA, Maribel ${ }^{1}$ \\ SÁNCHEZ - HERNÁNDEZ, Yusely J. ${ }^{2}$
}

\section{Resumen}

Esta investigación tiene como objetivo definir la estructura matricial de las competencias emprendedoras en el sector Cojines del Zaque, Tunja. Se usó una metodología descriptiva, de enfoque mixto y diseño de investigación acción. Los resultados definieron las correlaciones a partir de un análisis factorial, donde las habilidades culturales, artísticas, ciudadanas, autonomía y tratamiento de la información, explican en más de un $90 \%$ el modelo. Concluyendo que a través de la lúdica se fortalecen las competencias emprendedoras en los niños del sector.

Palabras clave: evaluación, competencia, emprendimiento, emprendegrama

\section{Summary}

This research aims to define the matrix structure of entrepreneurial skills in the cushion sector of Zaque Tunja. Using a descriptive methodology, of mixed approach and action research design. The results defined the correlations from a factor analysis. Where cultural, artistic, civic skills, autonomy and information processing explain the model in more than $90 \%$. Concluding that through play, the entrepreneurial skills of children in the sector are strengthened.

Keywords: evaluation, competition, entrepreneurship, entrepreneurship

\section{Introducción}

Las competencias emprendedoras desempeñan un papel fundamental en el avance económico y social de países desarrollados, en donde la educación emprendedora propicia habilidades y conocimientos cruciales para la generación de una mentalidad emprendedora y empresarial. El objetivo general de esta investigación fue evaluar en qué medida están relacionadas las dimensiones de la competencia emprendedora y competencias básicas propuestas en el emprendegrama, en la población de los cojines del zaque Tunja; mediante la definición de una estructura matricial para cada muestra motivo de estudio; en los objetivos específicos se estableció el diagnóstico socioeconómico de la población, participación ciudadana, diseño de estrategias pedagógicas para la identificación y evaluación de las competencias. Como soporte a la investigación y cumplimiento de los objetivos se desarrolla una revisión revisión de literatura.

\footnotetext{
${ }^{1}$ Docente Escuela de Ciencias Administrativas y Económicas, Investigadora y líder Grupo competitividad y Desarrollo Local CODEL categoría C. Universidad Pedagógica y Tecnológica de Colombia.maribel.martinez@uptc.edu.co

${ }^{2}$ Docente Escuela de Ciencias Administrativas y Económicas, Investigadora Grupo GICA categoría A. Universidad Pedagógica y Tecnológica de Colombia. yusely.sanchez@uptc.edu.co
} 


\subsection{Emprendimiento}

La sociedad actual experimenta cambios constantes que implican respuestas oportunas; es así como el emprendimiento como fenómeno multifacético se ha estudiado en las últimas décadas y ha vinculado a otras áreas de estudio como la psicología social, como alternativa de generación de procesos de innovación económica, cultural, social que brinda a las comunidades empoderamiento y opciones de aprovechamiento de sus recursos para mejorar sus condiciones actuales, contribuyendo al cambio del orden social en el que se insertan las personas (Gieure, Benavides-Espinosa, \& Roig-Dobón, 2020; Haugh \& Talwar, 2016 ; Toma, Grigore, \& Marinescu, 2014).

La Ley 1014 de 2006, en Colombia, busca propiciar la formación de estudiantes en competencias, habilidades y destrezas, que les permitan emprender iniciativas a nivel personal y profesional, en su doble sentido de acción de emprender y cualidad emprendedora, vinculadas al espíritu emprendedor o iniciativa empresarial, (Programa de las Naciones Unidas para el Desarrollo, PNUD), 2016). Por lo tanto, el emprendimiento se considera como medio para el desarrollo económico y social de los países, en donde las personas a partir de edades tempranas experimentan una serie de potenciales ámbitos incubadores, que contribuyen en distinto grado al desarrollo de aptitudes y capacidades emprendedoras (Kantis, 2017).

La tendencia de emprender está enlazada a la cultura y viceversa, convirtiéndose en actor que origina y promueve el espíritu emprendedor. Mientras, el que emprende debe impactar directamente y transformar su realidad; sus valores emprendedores deben ser una motivación interna, pero a la vez atada a la cultura y su entorno, generando un cambio a nivel extrínseco (Sánchez, Ward, Hernández, \& Florez, 2017). Además, si se forma a los niños para ser emprendedores, serán los responsables del futuro y propiciar los cambios necesarios para hacer del planeta un lugar mejor para vivir, y no esperar pasivamente a que el mundo sea diferente (Pico, 2012).

\subsection{Competencias emprendedoras}

Las habilidades y competencias, tienen su fundamento en la capacidad de las personas de hacer desde su condición neurofisiopsicológica; esta pueden ser específica, cuando es requerida en cierta tareas, e integrativa cuando se realiza en situaciones con mayor nivel de complejidad (Portillo-Torres, 2017). Estas competencias tienen como objetivo el desarrollo y consolidación de la cultura emprendedora, como estrategia de intervención socioeducativa encaminada a minimizar los riesgos del contexto de cada individuo. El hecho de emprender, supone un ejercicio de madurez, responsabilidad y autonomía. Desde el punto de vista social, implica conocimiento, manejo y participación de los actores en el territorio (Martínez \& Carmona, 2009). En el contexto de la cultura emprendedora son siete habilidades esenciales que se asocian con el espíritu emprendedor y empresarial, se dividen en dos fases: habilidades tempranas y habilidades de madurez. La primera involucra: creatividad y flexibilidad. La madurez consiste en habilidades de liderazgo, organización-planificación, motivación y control financiero (Ismail, Zain, \& Zulihar, 2015). Asimismo, las personas emprendedoras poseen otras habilidades como: iniciativa, innovación, responsabilidad, autonomía a la hora de tomar sus decisiones, asumen riesgos, se esfuerzan para conseguir sus objetivos y trabajan en equipo (Fernández, 2014). De igual manera, la actitud emprendedora puede entrenarse y potenciarse desde la enseñanza cualidades personales y habilidades sociales (Expertemprende, 2015), 
Para desarrollar las competencias necesarias para el emprendimiento, es necesario establecer y mantener redes de colaboración en investigación que potencialicen habilidades complementarias para participar en actividades complejas y riesgosas. Las redes socioeconómicas mejoran los lazos débiles y unen recursos para empoderar a los socialmente desfavorecidos para lograr la inclusión, reconocer y representar las necesidades, opiniones y valores (Deste, Mahdi, Neely, \& Rentocchini, 2012); (Karlidag-Dennis, Hazenberg, \& Dinh, 2020).

En el modelo de Cunha y Heckman, en el año 2007, las habilidades cognitivas y no cognitivas se desarrollan durante diferentes etapas de la vida, donde las habilidades aprendidas durante un período de la vida aumentan los beneficios de invertir en estas habilidades en períodos posteriores. Por lo tanto, las inversiones tempranas en habilidades pueden ser particularmente efectivas a largo plazo. Si se proporciona a los niños y jóvenes un panorama claro del proceso emprendedor, podría causar un efecto positivo en el sentido de que solo aquellos alumnos con alta capacidad emprendedora elegirán esta opción de vida. Por tanto, parece que las habilidades empresariales no cognitivas se desarrollan mejor a una edad temprana (Huber, Sloof, \& Van Praag, 2014).

\subsection{Educación para el emprendimiento}

La educación en emprendimiento es una disciplina con una fuerte aplicación e implica elevar el acto de formación de la personalidad del alumno. En estas condiciones las actividades educativas deben estar dirigidas a formación de habilidades específicas y cumplir con los requisitos del aprendizaje sostenible: utilizando métodos dinámicos familiarizándolos con aspectos económicos de la vida cotidiana, esto les ayuda a identificar y evaluar las consecuencias de las decisiones personales y cooperar con los padres y las autoridades, en un mundo donde existe una creciente interdependencia económica y social, la evaluación debe realizarse predominantemente como continua (Vilcov \& Dimitrescu, 2015).

La enseñanza en esta área emprendedora-empresarial tiene como objetivo ayudar a los estudiantes a adquirir habilidades y conocimientos, esenciales para el desarrollo de una mentalidad proactiva y emprendedora, lo cual implica la integración de planes de estudio, tutoría y asesoría. Es así como la educación fomenta la vocación y transforma futuros emprendedores en los activos de capital humano más valiosos de una organización, para aprovechar oportunidades y generar valor de acuerdo con las realidades y variedad de contextos (La Guardia, Gentile, Dal Grande, Ottaviano, \& Allegra, 2014; Gieure et al., 2020). Asimismo, cada uno de los niveles de educación deben servir de enlace con el mercado laboral para asegurar un mejor desempeño en un entorno cada vez más competitivo, en donde el estudiante cambia la forma de ver el mundo, independientemente de si eligen desarrollar su propio negocio o no. Por ello, es necesario desarrollar la capacidad para diseñar, gestionar y tener la mente emprendedora para la mejora continua (Benavides, Sánchez, \& Luna, 2010; Kabukcu, 2015).

La participación de los niños y jóvenes en las actividades educativas encaminadas al emprendimiento no es para la supervivencia económica del hogar, sino para prepararlos para que sean útiles para ellos mismos y para una sociedad que requiere personas con valores, actitudes proactivas, que generen cambios estableciendo una cultura emprendedora (Torimiro \& Dionco-Adetayo, 2005). Una cultura familiar con valores y recursos familiares fomenta la participación en procesos emprendedores para construir relaciones sociales que influyen en la intención y actitud empresarial. Las expectativas de prosperidad futura, son el resultado de tres combinaciones de factores: familiares - frugal, individual - mercado y familiar - interior, que muestran una realidad mucho más diversa (Kimmitt, Muñoz, \& Newbery, 2020; Elia, Margherita, \& Passiante, 2020). El número de emprendedores ha ido aumentando día a día en el mundo, a partir de las nuevas oportunidades, en consecuencia numerosos

estudios han abordado el tema de la determinación de los factores y competencias que afectan o influyen 
directamente con las intenciones emprendedoras de las personas y la relación entre la educación para el emprendimiento y las intenciones emprendedoras (Doğan, 2016).

La actual crisis social y económica revela cierta fragilidad entre los jóvenes cuando se trata de dinero y negocios. Por lo tanto, ser proactivo, con mentalidad enfocada en intentar siempre, nunca rendirse, debería ser un comportamiento habitual. Pero, lamentablemente, este no es el aspecto del mundo real. Los estudiantes aprenden mejor cuando hacen conexión entre aprendido y su aplicación. Por ende, necesitan desarrollar competencias que les animen a ser emprendedores y propicien el fortalecimiento de su potencial e inspirar su confianza y pasión empresarial. Finalmente, la educación para el emprendimiento debe ser el resultado de una estrategia colectiva en las escuelas, fuera de ellas y el gobierno nacional; y no el resultado de personas individuales (Tadeu \& Paiva, 2015; Cotoi, Bodoasca, Catana, \& Cotoi, 2011; Jena, 2020 ; Rosendahl Huber, Sloof, Van Praag, \& Parker, 2020).

\section{Metodología}

La metodología fue de tipo descriptivo. La investigación consistió en describir fenómenos, situaciones, contextos y sucesos; detallando cómo son y cómo se manifiestan pretendiendo medir o recoger información de manera independiente o conjunta sobre los conceptos y los efectos de las variables establecidas (Hernández Sampieri, Fernandez Collado, \& Baptista Lucio, 2010; Sampieri, 2014). Se utilizó con enfoque mixto y diseño de investigación acción participativa, la cual interactúa con distintos agentes para definir y dirigir sus propias necesidades, conflictos y soluciones orientadas a la transformación social de la comunidad, la cual permite hacer un análisis detallado al fenómeno a estudiar de acuerdo con las diversidades de contexto integrando saberes y aprendizajes de actores sociales e institucionales, que favorecen el diseño e implementación de estrategias, para dar respuesta a las problemáticas detectadas (Sigalat Signes, Calvo Palomares, Roig Merino, \& Buitrago Mera, 2019).

En este estudio se tomó una muestra 50 niños del sector Cojines de Zaque, Tunja, que a su vez, es el total de la población, los cuales se clasificaron en dos grupos, tomando como referencia lo establecido en el Departamento Administrativo Nacional de Estadística, DANE, que instaura los siguientes rangos de edad para cada nivel escolar: transición 5 años, primaria de 6 a 10 años, secundaria de 11 a 14 años y media de 15 a 16 años. En razón a estos criterios el primer grupo aglomera la población de 6 a 10 años y el segundo edades de 11 a 14 años; asimismo se definieron variables como estrato socioeconómico, edad y género.

El análisis de la información, se realizó partir de un análisis factorial de la correlación de cinco dimensiones de la capacidad emprendedora y ocho competencias básicas propuestas en el modelo que utiliza la escala de medición planteada por César García - Rincón de Castro (2013); posteriormente, se aplicaron pruebas de KMO y Bartlett, que determinaron los factores en su conjunto y su significancia, finalmente se realizó la segmentación de varimax en donde se asumió que los factores son independientes entre sí, de esta manera se minimizó el número de variables con altas cargas factoriales en el modelo de capacidad emprendedora.

\subsection{Métodos de recolección de información}

En la investigación se aplicaron los siguientes instrumentos de recolección de información:

Bitácora de trabajo, es un instrumento de registro y recolección de datos organizados para mantener un control sistemático de la información. El registro se llevó a cabo a través de un formato consignando los hallazgos más relevantes bajo criterios de: fecha, hora, lugar, rol, propósito del encuentro, dimensiones, aspectos formativos a nivel procedimental y afectivo-relacional, finalmente notas de campo con un sistema categorial (López \& González, 2014; Rekalde, Vizcarra, \& Macazaga, 2013). 
Las encuestas son medios eficientes para determinar la realidad sobre conocimientos, creencias, expectativas y comportamientos de las personas (Behar, 2008). Este instrumento fue aplicado a cincuenta (50) integrantes de la sector 3 de Tunja, manzana catastral 183 predio 6, denominación Cojines del Zaque, propietario Departamento de Boyacá, categoría de conservación según el POT predio con tratamiento de conservación monumental, con el objetivo de evaluar en qué medida están relacionadas las dimensiones de la competencia emprendedora y competencias básicas, según (César García - Rincón de Castro, 2013), como referencia del modelo conceptual para aprovechar alternativas que brinda el territorio ver gráfico 1.

Gráfico 1

Modelo conceptual y metodológico

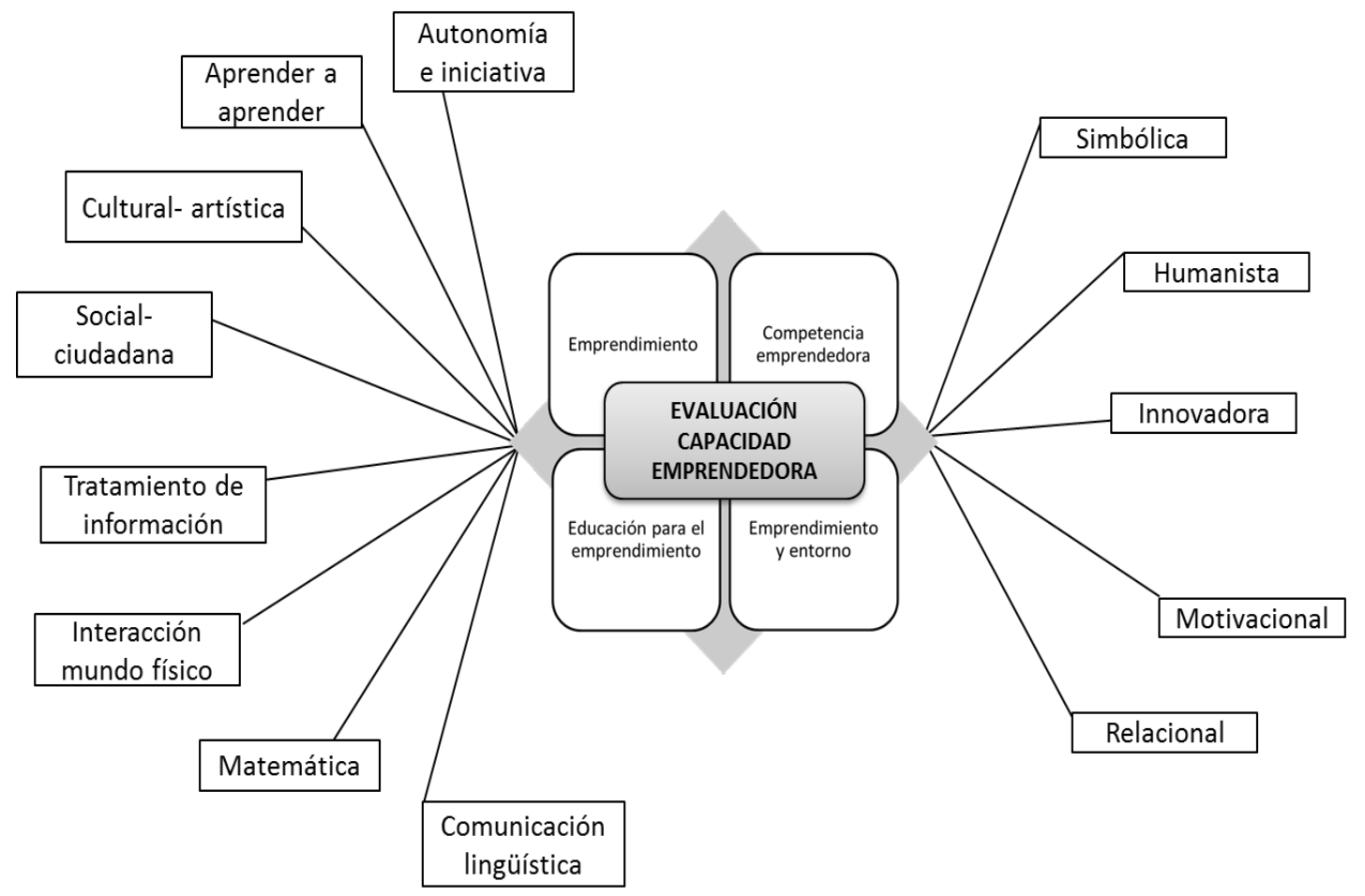

\section{Resultados}

Los resultados muestran la evaluación del desempeño de las competencias emprendedoras de acuerdo con las dimensiones relacional, motivacional, innovadora, humanista y simbólica; respecto a las competencias básicas de comunicación lingüística, matemática, conocimiento, interacción con el mundo físico, tratamiento de la información, social-ciudadana, cultural -artística, aprender a aprender y autonomía e iniciativa personal, para cada uno de los grupos establecidos, los datos son el resultado de la aplicación de los instrumentos: bitácora y encuesta.

\subsection{Bitácora}

La bitácora se elaboró durante los talleres participativos con los grupos establecidos. En la fase 1 se desarrolló actividad de reconocimiento del territorio con padres de familia, líderes sociales y comunidad a intervenir (niños y jóvenes), para identificar las condiciones sociales, económicas, culturales y recursos tangibles e intangibles disponibles. A continuación, en los cuadros 1 y 2 , se presenta la bitácora mencionada con información relevante recolectada a partir de la observación. 


\section{Cuadro 1}

Bitácora de observación grupo 1

\begin{tabular}{|c|c|c|}
\hline Lugar & Dimensión & Observaciones \\
\hline & Motivacional & $\begin{array}{l}\text { Son personas con relaciones interpersonales aceptables, con procesos de comunicación inicialmente limitada, sin } \\
\text { embargo durante el transcurso se evidenció fortalecimiento a medida que los talleres avanzaron generando lazos de } \\
\text { cooperación entre compañeros y talleristas. Expresarse con sencillez, claridad y veracidad es una competencia } \\
\text { representativa en el grupo, tienden a entender algunas situaciones que son incorrectas para no imitarlas, no tienen } \\
\text { las herramientas mentales suficientes para soportar los procesos de aplicación de valores, se presenta liderazgo } \\
\text { especialmente del género femenino. } \\
\text { Por la edad de los participantes las actividades lúdicas generaron altos niveles de motivación de logro, emociones } \\
\text { proactivas, espontáneas y autonomía personal, para diferenciarse del grupo y alcanzar reconocimiento al afrontar } \\
\text { retos. } \\
\text { La creatividad e innovación en los niños, es importante para su desarrollo integral, presentaron altos niveles de } \\
\text { independencia en su forma de pensar y actuar, resultado de la aplicación de las estrategias pedagógicas; su grado de } \\
\text { sensibilidad frente al entorno es relevante siendo secuela de las condiciones precarias de muchos de ellos, } \\
\text { obligándolos a entender y generar escudos o protecciones, frente a situaciones de riesgo. }\end{array}$ \\
\hline \multirow[t]{2}{*}{ Cojines del Zaque } & Humanista & $\begin{array}{l}\text { Esta dimensión se ve altamente influenciada por el desarrollo de competencias y valores, tales como el respeto, } \\
\text { responsabilidad, en cada encuentro o taller este grupo de niños, se caracterizaron por su puntualidad y sostenibilidad } \\
\text { en las actividades. Igualmente se destaca toma de conciencia de las necesidades del otro, deseo de contribuir y } \\
\text { colaborar para su satisfacción personal y colectiva, especialmente en los talleres de estilos de vida saludable, en los } \\
\text { cuales los más pequeños ayudaron a los demás en la ejecución de las dinámicas reconociendo la importancia de la } \\
\text { temática con sentido proactivo, compromiso y transferencia de conocimientos en sus hogares, aspecto que impactó } \\
\text { en época de pandemia de forma positiva. }\end{array}$ \\
\hline & Simbólica & $\begin{array}{l}\text { Los resultados de esta dimensión, demuestran óptimo nivel de integración de las estrategias pedagógicas diseñadas } \\
\text { e implementadas para los componentes artísticos - sociales. La competencia más notable fue la simpatía entendida } \\
\text { como comprender sentimientos y emociones, fue expresada a través de dibujos en el desarrollo de los talleres de } \\
\text { cartografía social, los cuales evidencian una capacidad de ponerse en el lugar del otro, al comprender las realidades } \\
\text { y dificultades de sus compañeros y habitantes permanentes del mismo territorio, permitiendo generar de forma } \\
\text { espontánea sentimientos de tristeza, alegría, miedo, ira. La locuacidad es limitada en algunos participantes, } \\
\text { especialmente en las edades más cortas, generando para el grupo de orientadores casos únicos motivos de estudio y } \\
\text { seguimiento. }\end{array}$ \\
\hline
\end{tabular}

Cuadro 2

Bitácora de observación grupo 2

\begin{tabular}{|c|c|c|}
\hline Lugar & Dimensión & Observaciones \\
\hline & Relacional & $\begin{array}{l}\text { Son personas con relaciones interpersonales complejas, los procesos de comunicación presentaron dificultades por } \\
\text { los rangos de edades y la tipología de las actividades, las cuales requerían alto grado de concentración e innovación } \\
\text { durante el proceso. Se evidenció el paso de la resistencia al reconocimiento de la importancia e impacto a corto y } \\
\text { largo plazo en el desarrollo de estas competencias. Asimismo, expresarse con claridad es una competencia } \\
\text { representativa en el grupo, tienden a no entender algunas situaciones que son incorrectas, tienen herramientas } \\
\text { mentales complejas e influenciables por su entorno requiriendo mayores esfuerzos para reforzar valores. Se presenta } \\
\text { liderazgo especialmente del género masculino. }\end{array}$ \\
\hline & Motivacional & $\begin{array}{l}\text { Los participantes presentan niveles de motivación de logro y espontaneidad limitados, al considerar que expresar sus } \\
\text { emociones libremente, puede ser muestra de debilidad o de crítica por parte del entorno. La autonomía y } \\
\text { reconocimiento al afrontar retos, es un factor diferenciador altamente influenciado por su entorno, dificultades y } \\
\text { realidades. }\end{array}$ \\
\hline \multirow[t]{3}{*}{ Cojines del Zaque } & Innovadora & $\begin{array}{l}\text { La innovación y creatividad, es un componente que presenta contradicción, solo se ve desarrollado siempre y cuando } \\
\text { este mediado por TIC, no reconocen procesos de inducción e importancia de conocer previamente conceptos, } \\
\text { procesos y procedimientos esenciales. Desarrollan su sentido de aprendizaje a través de la intuición y práctica, siendo } \\
\text { dominante este aspecto; su grado de sensibilidad es muy alto frente al entorno reconociendo y rechazando sus } \\
\text { condiciones vulnerables, generando barreras frente a situaciones de riesgo. }\end{array}$ \\
\hline & Humanista & $\begin{array}{l}\text { Las variables asociadas al respeto y responsabilidad, evidenciaron impuntualidad y actitudes desfavorables en las } \\
\text { actividades. El deseo de contribuir y colaborar para su satisfacción personal fue alto, mientras la colectiva es casi nula, } \\
\text { se caracterizaron los participantes en sus aspectos humanos, mostraron bajos niveles de sensibilidad en algunas } \\
\text { ocasiones y actitud moderada frente a las problemáticas y capacidad de resolución. }\end{array}$ \\
\hline & Simbólica & $\begin{array}{l}\text { La dimensión artística - social, muestra en los participantes sentimientos y emociones limitadas por factores del } \\
\text { entorno. Las actividades que implican ponerse en el lugar del otro, presentan bajos niveles de desarrollo, prefieren } \\
\text { trabajar de forma individual. Los sentimientos de miedo e ira, se manifiestan con frecuencia. El territorio se ha } \\
\text { convertido en aspecto simbólico y de afirmación de sus condiciones; sin embargo se muestra un porcentaje } \\
\text { considerable de jóvenes después de las actividades realizadas que pueden ser líderes y empoderase, a partir del } \\
\text { aprovechamiento de las potencialidades y alianzas conformadas en los últimos años, para aunar esfuerzos } \\
\text { económicos, investigación, extensión y proyección social con instituciones (universidades, gobierno, entes sociales), } \\
\text { como apoyo al fortalecimiento de capacidades. }\end{array}$ \\
\hline
\end{tabular}




\subsection{Análisis de la encuesta}

Los resultados del análisis e interpretación de la encuesta para la evaluación de las dimensiones de la competencia emprendedora y competencias básicas, está desarrollada bajo el modelo propuesto por (César García - Rincón de Castro, 2013).

\section{Análisis factorial}

Análisis de tipo multivariante de interdependencia en el cual se encuentra un número más pequeño de factores no observables, que, perdiendo el mínimo de información, expresan las relaciones establecidas entre las variables observadas (Guti, 2019). Con esta técnica estadística se evaluó el modelo de capacidad emprendedora, en un población especifica ubicada en el territorio denominado Cojines del Zaque considerado como patrimonio cultural e histórico de la región central del país, hallando grupos homogéneos de variables a partir de un emprendegrama, que contiene cinco dimensiones y ocho competencias básicas, esenciales a desarrollar durante los procesos emprendedores que configuran la competencia emprendedora y sus valores asociados. Esta evaluación del modelo se realizó con población en edad escolar de acuerdo con lo establecido por el Ministerio de Educación, registrando que el 44\% los niños están en edades de 6-11 años y 56\% en edades de 11-14 años, en donde la estructura poblacional para la variable genero representa $42 \%$ masculino y $58 \%$ femenino; a su vez la variable de socioeconómica se presentó $62 \%$ en estrato 1 y $38 \%$ en estrato 2 , ubicados en la zona de influencia del patrimonio histórico denominado cojines del Zaque (ver gráfico 2). Los factores analizados en el estudio son producto de una combinación lineal de variables internas correlacionadas, con el fin de reducir datos en un número pequeño de factores, que sean capaces de explicar la mayor proporción de la variabilidad total del modelo.

\section{Gráfico 2}

Estructura poblacional muestra
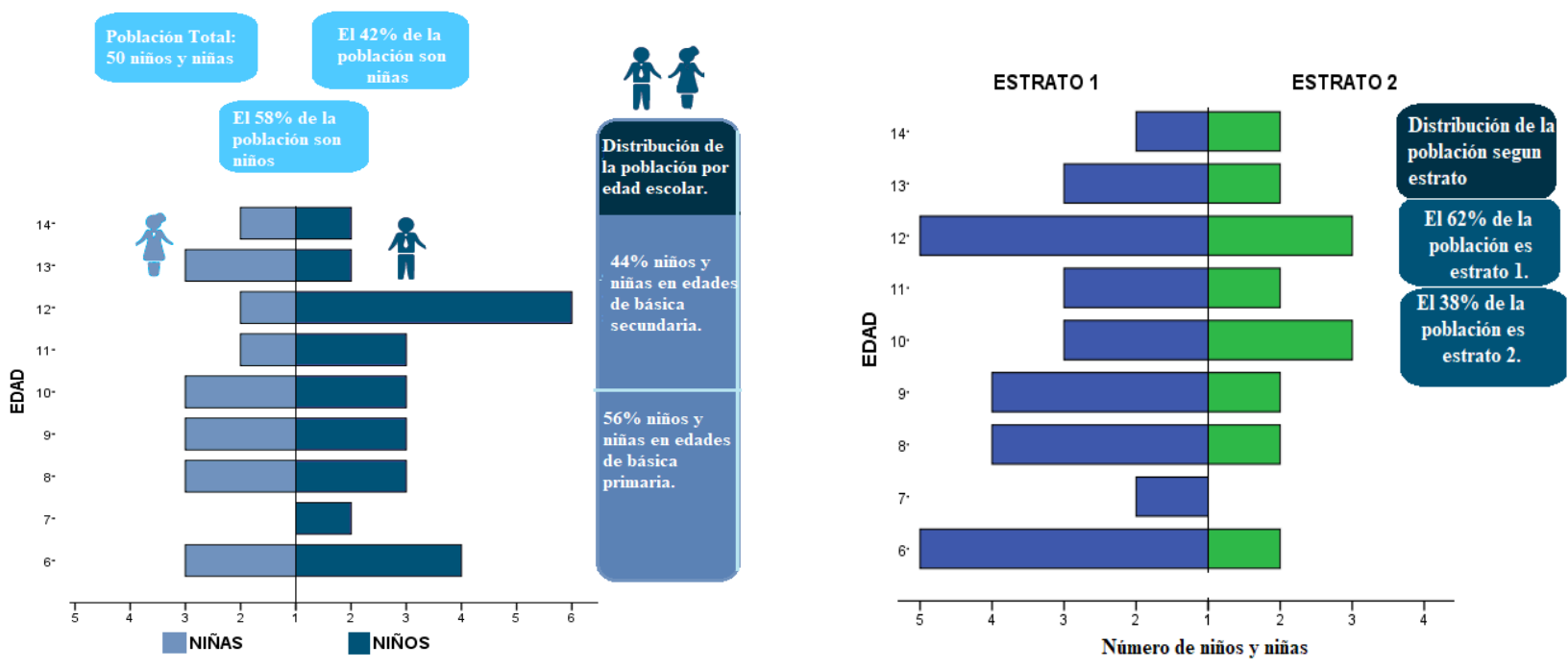

Fuente: SPSS Statistics

\section{Matriz de correlaciones}


La matriz de correlaciones para modelo de capacidad emprendedora grupo 1, generada a partir de los resultados de la segmentación por edades y nivel de dominancia de las competencias, muestra en el aspecto social y ciudadano, un nivel muy alto de conocimiento de sí mismos y reconocimiento de su rol en el entorno que representa influencia directa de forma negativa, al considerarse como un sector de alta vulnerabilidad, baja seguridad y abandono por parte del gobierno municipal y departamental, los cuales por la particularidad del territorio es difícil determinar su responsabilidad, ocasionando niveles insuficientes de inversión económica y social, obligado a forjar habilidades para participar activa y plenamente en la vida cívica, en pro de la consecución de apoyo para fortalecer los diferentes ámbitos de la población y satisfacer las necesidades básicas de la misma; siendo así modelos a seguir de líderes sociales y promotores de su cultura para los más jóvenes, ver cuadro 3.

Cuadro 3

Matriz de correlaciones modelo de capacidad emprendedora grupo 1

\begin{tabular}{|c|c|c|c|}
\hline \multicolumn{4}{|c|}{ Competencia social y ciudadana } \\
\hline Dimensión & Correlación & Significancia & Conclusión \\
\hline Relacional & 0,923 & 0,002 & $\begin{array}{l}\text { Suscita la interacción con otras personas, generando e interiorizando } \\
\text { cultura. }\end{array}$ \\
\hline Motivacional & 0,821 & 0,001 & Promueve la capacidad y necesidad de interacción afectiva con semejantes \\
\hline Innovadora & 0,752 & 0,003 & $\begin{array}{l}\text { Crea una diversidad de aspectos que permiten a la persona interactuar con } \\
\text { otras personas. }\end{array}$ \\
\hline Humanista & 0,873 & 0,001 & $\begin{array}{l}\text { Favorece la adaptación al medio, lo que hace a la persona ser ella misma, } \\
\text { a partir de su contexto económico, social y cultural. }\end{array}$ \\
\hline Simbólica & 0,913 & 0,003 & $\begin{array}{l}\text { Beneficia la construcción de la propia identidad, es otro de los procesos } \\
\text { sociales básicos. }\end{array}$ \\
\hline
\end{tabular}

Fuente: SPSS Statistics

La competencia cultural y artística, presenta mayor nivel de correlación en las dimensiones relacional con 0,933 y simbólica 0,918 como se evidencia en el cuadro 4, como resultado del desarrollo de establecimiento de relaciones humanas de calidad y mecanismos de fortalecimiento de las habilidades comunicativas como medio para expresar actitudes y valores, necesarios para dar a conocer al mundo que su territorio no solo es un lugar que puede causar daño y acciones de rechazo, sino un lugar que hace muchos siglos fue considerado cuna de asentamientos indígenas, que guarda la memoria del desarrollo histórico con la imborrable huella dejada en los vestigios indígenas y las construcciones producto del proceso de conquista y colonización (Gobernación de Boyacá, 2011). Por lo tanto, se encaminó la estrategia pedagógica asumiendo la tarea de abordar los hechos artísticos desde esta perspectiva y no sólo desde los valores formales y estéticos, contribuyendo así al fortalecimiento de la competencia.

Cuadro 4

Matriz de correlaciones modelo de capacidad emprendedora grupo 1

\begin{tabular}{|c|c|c|c|}
\hline \multicolumn{4}{|c|}{ Competencia cultural y artística } \\
\hline Dimensión & Correlación & Significancia & Conclusión \\
\hline Relacional & 0,933 & 0,002 & $\begin{array}{l}\text { Entiende lenguajes artísticos como recursos de expresión y comunicación, } \\
\text { consigo mismo y con el entorno. }\end{array}$ \\
\hline Motivacional & 0,811 & 0,001 & $\begin{array}{l}\text { Crea productos artísticos de manera personal y motivada, como estrategia } \\
\text { de expresión, representación de emociones y contornos de vida. }\end{array}$ \\
\hline Innovadora & 0,722 & 0,003 & $\begin{array}{l}\text { Las manifestaciones culturales comprenden la evolución del pensamiento, } \\
\text { corrientes, modas y gustos, representando la vida cotidiana de las } \\
\text { personas y de las sociedades, en su entorno. }\end{array}$ \\
\hline Humanista & 0,853 & 0,001 & $\begin{array}{l}\text { La sostenibilidad, transparencia, solidaridad, son factores representativos } \\
\text { en las edades del grupo estudiado, la conciencia de las necesidades de su } \\
\text { entorno y la voluntad de ayudar para cubrir esas necesidades, así como el } \\
\text { valor de la familia. }\end{array}$ \\
\hline Simbólica & 0,918 & 0,003 & $\begin{array}{l}\text { Valoran de manera reflexiva y crítica los elementos que integran el } \\
\text { patrimonio artístico y cultural, de su territorio como fundamento de la } \\
\text { identidad intercambio y diálogo de costumbres. }\end{array}$ \\
\hline
\end{tabular}

Fuente: SPSS Statistics 
En las dimensiones relacional y simbólica con un 0.956, se ve representada la correlación más alta en la competencia de autonomía e iniciativa, en el grupo 1 se visualiza en los participantes un enfoque estratégico de retos y oportunidades a los que se tiene que enfrentar a lo largo de su vida, generando habilidades en aspectos de toma de decisiones, perseverancia, sin embargo algunos presentan bajos niveles de autoestima. Igualmente al hacer frente a un proyecto creativo actúan con autonomía, ponen en marcha iniciativas para aprovechar oportunidades con soluciones innovadoras. La expresión artística, emite respuestas abiertas con diferentes opciones personales y colectivas, permitiendo la afirmación de la autoestima e identidad, trabajando con las emociones, afectividad y gestión de respuestas a las realidades del territorio. Asimismo, las áreas y materias de la competencia artística contribuyen a la adquisición de la conciencia, gestión, control de decisiones y balance de los aprendizajes que realiza (ver cuadro 5).

\section{Cuadro 5}

Matriz de correlaciones modelo de capacidad emprendedora grupo 1

\begin{tabular}{|c|c|c|c|}
\hline \multicolumn{4}{|c|}{ Competencia autonomía e iniciativa personal } \\
\hline Dimensión & Correlación & Significancia & Conclusión \\
\hline Relacional & 0,919 & 0,002 & $\begin{array}{l}\text { Las habilidades de liderazgo en los niños son una composición de } \\
\text { características personales y actitudinales, que permiten desarrollar } \\
\text { capacidades y habilidades para idear y construir; en beneficio de su } \\
\text { comunidad. }\end{array}$ \\
\hline Motivacional & 0,721 & 0,001 & $\begin{array}{l}\text { La humildad es uno de los valores esenciales en la educación de los niños, } \\
\text { se evidencia en el respeto hacia los demás, sin importar su edad, estrato, } \\
\text { y rol; valora al otro por el aporte en su vida, siendo promovido por su } \\
\text { actitud permanente de aprendizaje. }\end{array}$ \\
\hline Innovadora & 0,852 & 0,003 & $\begin{array}{l}\text { El desarrollo creativo presenta momentos de mutisensoriales presente } \\
\text { en la actividad artística (música), momentos simbólicos y representativos } \\
\text { evidencia de su contexto cultural y patrimonial, y finalmente una etapa } \\
\text { intuitiva causada por los altos niveles de curiosidad natural de querer } \\
\text { saber algo mediante la exploración y aprendizaje que estimulan el } \\
\text { crecimiento personal. }\end{array}$ \\
\hline Humanista & 0,903 & 0,001 & $\begin{array}{l}\text { El respeto a sí mismos y a los demás; enfocados al bien común como } \\
\text { principio básico para convivir en comunidad, mostrando consideración de } \\
\text { las necesidades y expectativas de los demás, en determinadas situaciones, } \\
\text { contextos y creencias; procurando no causar un perjuicio. Asimismo el } \\
\text { compromiso, como valor implica una relación directa con la autonomía y } \\
\text { perseverancia en la toma de sus propias decisiones; y reconocimiento de } \\
\text { estas como consecuencia de sus conductas. }\end{array}$ \\
\hline Simbólica & 0,993 & 0,003 & $\begin{array}{l}\text { En la dimensión simbólica, se muestra la capacidad de expresar las ideas } \\
\text { en varios formatos comunicativos, implica la necesidad de la simpatía, } \\
\text { como una expresión afectiva que existe entre dos o más personas de } \\
\text { manera espontánea, forma parte de la personalidad y promueve las } \\
\text { relaciones interpersonales de forma positiva, apoyando la expresión de } \\
\text { pensamientos tanto verbal como no verbal de necesidades, deseos y } \\
\text { sentimientos. }\end{array}$ \\
\hline
\end{tabular}

Fuente: SPSS Statistics

Para el grupo 2 la correlación más representativa está dada en las dimensiones simbólica 0,993 y relacional 0,919 ( ver cuadro 6), las cuales presentan mayor nivel de desempeño de la población en edad escolar de 11 a 14 años en la competencia de conocimiento e interacción con el mundo físico, la muestra se desenvuelve de forma autónoma, facilitando el análisis e interpretación de las condiciones reales, grupos de interés y procesos de comunicación con el medio, permitiendo el reconocimiento de su rol en el contexto patrimonial e histórico, adoptando una disposición proactiva que facilita el aprovechamiento de capacidades presentes en el territorio y su interpretación; lo que exige la aplicación de los conceptos y principios básicos que permiten el análisis de los fenómenos sociales, económicos, políticos, para la toma de decisiones como elemento clave para el mejoramiento de la calidad de vida de las personas y el cumplimiento de los objetivos trazados. 
Cuadro 6

Matriz de correlaciones modelo de capacidad emprendedora grupo 2

\begin{tabular}{cccl}
\hline Dimensión & \multicolumn{3}{c}{ Competencia: conocimiento e interacción con el mundo físico } \\
\hline Relacional & 0,919 & Significancia & \multicolumn{1}{c}{ Conclusión } \\
\hline Motivacional & 0,881 & 0,002 & $\begin{array}{l}\text { Sitúa y relaciona los espacios con el cumplimiento de los objetivos trazados } \\
\text { en las diferentes actividades. } \\
\text { La población motivo del estudio, evalúa y selecciona el mejor medio } \\
\text { posible para el cumplimiento de las metas trazadas. }\end{array}$ \\
Innovadora & 0,001 & $\begin{array}{l}\text { La población motivo del estudio recrea e interrelaciona los medios actores } \\
\text { y elementos disponibles para la construcción de productos y desarrollo de } \\
\text { actividades de forma creativa e innovadora. }\end{array}$ \\
Bumanista & 0,003 & $\begin{array}{l}\text { Busca y diseña espacios a partir de la observación de su entorno } \\
\text { económico, patrimonial y ambiental. Encaminado al mejoramiento de las } \\
\text { condiciones de vida actual- } \\
\text { Genera procesos de comunicación con el medio, visualizando su entorno } \\
\text { en condiciones actuales que permiten el reconocimiento de su rol en el } \\
\text { contexto patrimonial e histórico. }\end{array}$ \\
\hline
\end{tabular}

Fuente: SPSS Statistics

Tratamiento de la información y competencia digital, esta competencia se refiere a la gestión del conocimiento en las áreas de: creación, seguridad, información, comunicación y transferencia, se evidenció que el uso de los recursos tecnológicos puede resolver problemas del contexto de modo eficiente, haciendo uso de recursos digitales y mediáticos para trabajar de desarrollar proyectos de forma colaborativa y responsable. Asimismo, la tecnología sirvió como un medio de empoderamiento para sus vidas, y no simplemente como una forma de entretenimiento y ocio (ver cuadro 7).

Cuadro 7

Matriz de correlaciones modelo de capacidad emprendedora grupo 2

\begin{tabular}{cccc}
\hline & \multicolumn{2}{c}{ Competencia: tratamiento de la información y competencia digital } \\
\hline Dimensión & Correlación & Significancia & \\
\hline Relacional & 0,854 & 0,03 & Potencia relaciones y redes con su entorno familiar y escolar. \\
Motivacional & 0,954 & 0,01 & Evidencia y optimiza logros y proyectos a través del uso de la tecnología. \\
Innovadora & 0,721 & 0,0031 & $\begin{array}{l}\text { Genera nuevos procesos para la aplicación herramientas informáticas y } \\
\text { materiales disponibles en su entorno. }\end{array}$ \\
Humanista & 0,932 & 0,0012 & $\begin{array}{l}\text { Propicia espacios para la generación de relacionamiento con personas que } \\
\text { permitan dar a conocer el valor del patrimonio histórico de su comunidad. }\end{array}$ \\
Simbólica & 0,833 & 0,011 & Sirve para comunicarse más rápido y de forma eficiente haciendo uso de \\
& & & herramientas tecnológicas. (redes sociales)
\end{tabular}

Fuente: SPSS Statistics

\section{Prueba de KMO y BARTLETT}

Para el modelo la prueba $\mathrm{KMO} \geq 0.849$, indica que es apropiado aplicar el análisis factorial a la matriz de datos bajo estudio del modelo de evaluación de competencias emprendedoras y la muestra tomada para el estudio es apropiada, por lo tanto se puede continuar con la aplicación del análisis factorial.

\section{Segmentación varimax: extracción de los factores iniciales y necesarios que representen a los datos originales}

Se utilizó el método de segmentación varimax, donde se asume que los factores son independientes entre sí de esta manera minimizamos el número de variables con altas cargas factoriales, en un factor haciendo que la matriz de componentes sea más fácil de interpretar. Para el modelo de capacidad emprendedora desarrollado en el grupo de edades 6-11 años identificado como grupo (1), muestra tres factores de mayor influencia en el modelo, estas competencias y habilidades están encaminadas en aspectos: social-ciudadano, cultural-artístico, autonomía-iniciativa personal. Para el grupo (2) el modelo arrojo dos factores, que generan mayor nivel de 
dominancia estas competencias están relacionadas con el conocimiento e interacción con el mundo físico y tratamiento de la información y competencia digital (ver gráfico 2).

Gráfico 2

Sedimentación grupo 1 y 2

a) Sedimentación edades 6-10 años

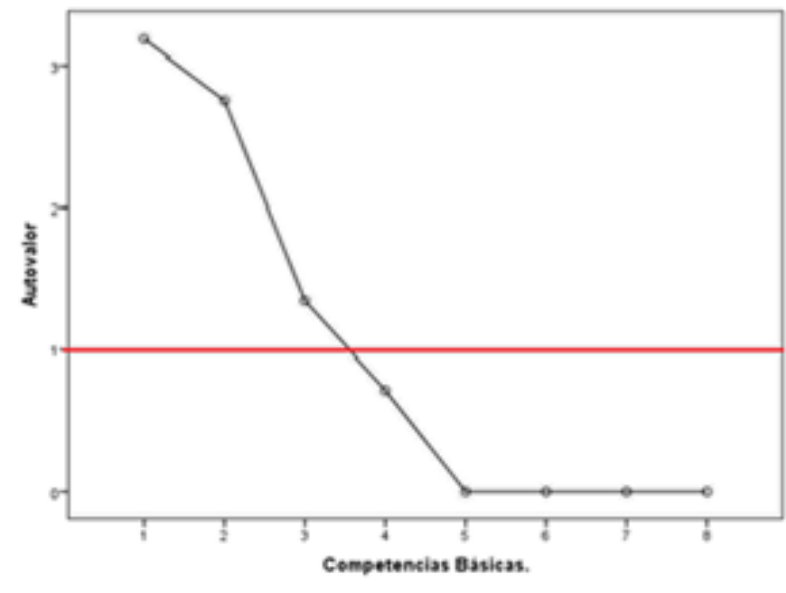

b) Sedimentación edades 11-14 años

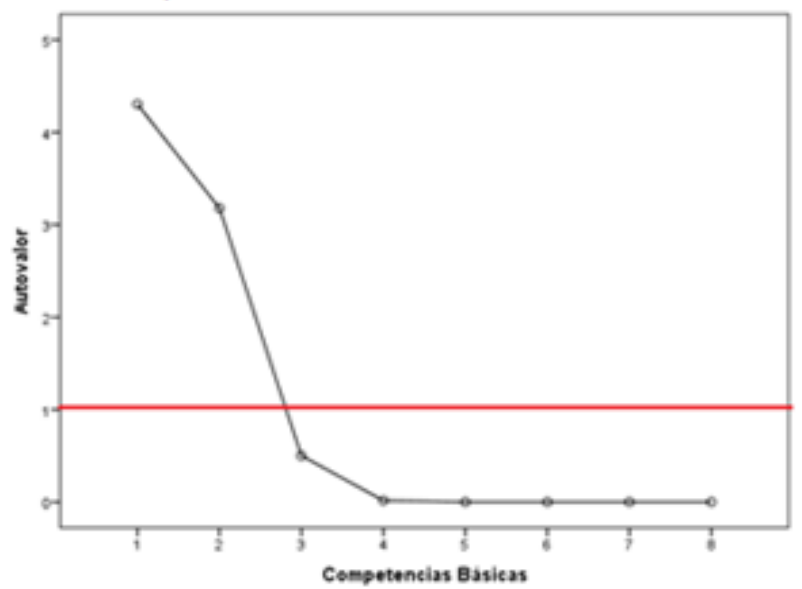

Fuente: SPSS Statistics

\section{Comunalidades}

Para el modelo de capacidad emprendedora, en el cuadro 8 y 9 se observa las comunalidades asignadas a las variables (preliminar) y las reproducidas por el medio factorial (extracción). La comunalidad de una variable, es la proporción de su varianza que puede ser explicada por el modelo factorial obtenido (Cuervo, Vázquez, Acuña, Murrieta, \& Quiñonez, 2020), en el cuadro 8 para el grupo 1, la variable con menor explicación en el modelo es la comunicación lingüística con un ,717 y los componentes principales que mejor explican el modelo con valores superiores a 0,9 y son capaces de reproducir su variabilidad original son: social ciudadana, cultural artística y Autonomía e Iniciativa. El cuadro 9, muestra la variable con menor explicación es la autonomía e iniciativa con un ,707 y los componentes principales que mejor explican el modelo con valores superiores a 0,9 y son capaces de reproducir su variabilidad original son: Competencia en el conocimiento y la interacción con el mundo físico como la habilidad para desenvolverse de forma autónoma en distintos ámbitos y tratamiento de la información y competencia digital entendida como la capacidad de buscar, procesar, comunicar y transformarla la información en conocimiento. Para cada grupo en estudio el número de factores obtenidos es suficiente para explicar todas y cada una de las variables incluidas; no se excluye ningún factor por que el valor generado a partir de la extracción que es superior a ,5.

\section{Cuadro 8}

Comunalidades capacidad emprendedora grupo 1

\begin{tabular}{lcc}
\hline \multicolumn{1}{c}{ Competencia } & Inicial & Extracción \\
\hline Comunicación Lingüística & 1,000 &, 717 \\
Lógico matemática & 1,000 &, 931 \\
Interacción con el medio & 1,000 &, 914 \\
Información digital & 1,000 &, 931 \\
Social Ciudadana & 1,000 &, $984^{*}$ \\
Cultural Artística & 1,000 &, $939^{*}$ \\
Aprende a aprender & 1,000 &, 890 \\
Autonomía e Iniciativa & 1,000 &, $956^{*}$ \\
\hline
\end{tabular}

Fuente: SPSS Statistics 
Cuadro 9

Comunalidades capacidad emprendedora grupo 2

\begin{tabular}{lcc}
\hline \multicolumn{1}{c}{ Competencia } & Inicial & Extracción \\
\hline Comunicación Lingüística & 1,000 &, 806 \\
Lógico matemática & 1,000 &, 831 \\
Interacción con el medio & 1,000 &, $957^{*}$ \\
Información digital & 1,000 &, $947^{*}$ \\
Social Ciudadana & 1,000 &, 809 \\
Cultural Artística & 1,000 &, 839 \\
Aprende a aprender & 1,000 &, 890 \\
Autonomía e Iniciativa & 1,000 &, 707 \\
\hline
\end{tabular}

Fuente: SPSS Statistics

El cuadro de porcentajes de varianza explicada (ver cuadro 8-9) presenta los auto- valores de la matriz de varianzas-covarianzas y del porcentaje que representa cada uno de ellos. El autovalor que expresan mayor cantidad de varianza total explicada para el grupo 1, es el componente social y ciudadana con un $39,758 \%$, este incluye el conocerse y valorarse, comunicación en contextos diversos y su relación como ciudadano de un territorio. En el caso del grupo 2 analizado existen tres autovalores mayores que 1 y dos para el otro grupo analizado, como resultado del procedimiento de extracción se explica en un 91,001\% y 93,528 de la varianza de los datos originales para cada grupo respectivamente.

\section{Cuadro 10}

Varianza total explicada grupo 1

\begin{tabular}{ccccc}
\hline & \multicolumn{4}{c}{ Sumas de extracción de cargas al cuadrado } \\
\cline { 2 - 5 } Componente/competencia & Total & Total & \% de varianza & $\%$ acumulado \\
\hline Social Ciudadana & 3,181 & 3,181 & 39,758 & 39,758 \\
Cultural Artística & 2,754 & 2,754 & 34,430 & 74,188 \\
Autonomía e Iniciativa & 1,345 & 1,345 & 16,813 & 91,001 \\
\hline
\end{tabular}

Fuente: SPSS Statistics

Cuadro 11

Varianza total explicada grupo 2

\begin{tabular}{lccc}
\hline \multirow{2}{*}{ Componente } & \multicolumn{3}{c}{ Sumas de extracción de cargas al cuadrado } \\
\cline { 2 - 4 } & Total & \% de varianza & \% acumulado \\
\hline Interacción con el medio & 4,303 & 53,794 & 53,794 \\
Información digital & 3,179 & 39,735 & 93,528 \\
\hline
\end{tabular}

Fuente: SPSS Statistics

\section{Discusión y Conclusiones}

La discusión se aborda a partir de la triangulación de los datos e integración de los principales hallazgos en la aplicación de los instrumentos utilizados en la investigación, las dimensiones, competencias establecidas y la relevancia con relación al objetivo trazado.

La población en estudio posee competencias como: iniciativa, creatividad, son capaces de innovar, actúan con autonomía a la hora de tomar sus decisiones, asumen riesgos y son responsables de sus acciones. Además, los grupos estudiados se esfuerzan para conseguir sus objetivos y son capaces de trabajar en equipo. Las habilidades 
tienen su soporte en la capacidad del individuo, de hacer desde sus condiciones neurofisiopsicológicas; estas pueden ser específicas, cuando son requeridas en ciertos trabajos, e integrativas en situaciones complejas.

En las dimensiones motivacional, innovadora, humanista y simbólica, para el grupo 1, las competencias socialciudadana; cultural-artística y autonomía e iniciativa, explican el modelo en un 91,001\%, presentando correlación positiva significativa en las intenciones y habilidades emprendedoras. El estudio encontró una correlación entre la educación e intención emprendedora, lo que confirma el importante papel que desempeña la educación. Además, se reveló que los participantes con padres autónomos y que apoyaron de manera decidida este proceso presentaron mayores intenciones emprendedoras que los otros. Este resultado muestra la transcendencia de la familia y su entorno, como modelo a seguir. Por lo tanto, existe una necesidad de regular las prácticas de educación (Doğan, 2016; Portillo-Torres, 2017; Martínez \& Carmona, 2009; Fernández, 2014).

Igualmente, los participantes de este grupo, como resultado de las actividades lúdicas generaron altos niveles de motivación de logro, emociones proactivas, espontáneas, autonomía personal y reconocimiento al afrontar retos. Tambien, es importante para el desarrollo integral, fortalecer habilidades y competencias de forma atrayente, evidenciando el progreso de las competencias a través de la lúdica aplicada, las competencias tienden a ser más específicas y los participantes poseen mayores criterios para elegir de acuerdo con sus preferencias y habilidades (Mateus \& Galeano, 2016). Cabe señalar que las relaciones interpersonales son aceptables, con procesos de comunicación inicialmente limitados, tienden a entender algunas situaciones de acuerdo con el grado de impacto negativo o positivo, se presenta liderazgo especialmente del género femenino, las habilidades de liderazgo en la vida temprana predicen las ganancias en la vida posterior, notablemente. No obstante, el ascenso a niveles superiores de liderazgo no depende únicamente de las oportunidades de desarrollo prescritas, sino que depende de las formas en que ciertos grupos son excluidos sistemáticamente del capital social y cultural, desde muy temprano en sus vidas (Fitzsimmons \& Callan, 2020;Santos, Marques, \& Ferreira, 2020). Como consecuencia se muestra un grado de sensibilidad importante frente al entorno que es relevante siendo secuela de las condiciones precarias de muchos de ellos, obligándolos a entender y generar escudos o protecciones frente a situaciones de riesgo. Finalmente, para este grupo, los resultados demuestran el nivel de integración de las estrategias pedagógicas diseñadas e implementadas para los componentes establecidos.

Con respecto al grupo 2 en estudio, los hallazgos más relevantes están relacionados con las competencias de interacción con el medio, tratamiento de la información y habilidades digitales, influenciadas altamente por relaciones interpersonales complejas y procesos de comunicación rangos particulares propios de edades. Igualmente los jóvenes se expresan con claridad, presentan herramientas mentales complejas, liderazgo especialmente del género masculino. Además la motivación al logro y espontaneidad son limitadas, la autonomía y reconocimiento al afrontar retos, es un factor diferenciador altamente afectado por sus realidades. Del mismo modo, la innovación y creatividad son componentes que presentan contradicción, solo se desarrollan cuando están mediados por las TIC. No reconocen procesos de inducción e importancia de conocer previamente conceptos y procedimientos esenciales; desarrollan su sentido de aprendizaje a través de la intuición y práctica, siendo dominante este aspecto. Las competencias digitales constituyen la generación de actitudes, habilidades y destrezas con relación a la apropiación de la información construyendo expectativas sobre el uso y apropiación en el desarrollo de habilidades transversales en el proceso, ejecución actividades, prácticas escolares y extracurriculares; es así como las TIC promueven diferentes formas de optimizar el tiempo en labores diarias, laborales, académicas y emprendedoras, mediante el manejo de los recursos digitales, generando una percepción positiva como fuente valiosa, para adquirir conocimiento y facilitando del trabajo colaborativo (Bonilla-del-río, Diego-Mantecón, \& Lena-Acebo, 2018; Ruiz \& Uribe, 2013;Martinez, 2018). En este mundo cada vez más complejo e interconectado, ya no es posible acudir a soluciones normalizadas, la necesidad de nuevas formas de solucionar problemas crece al mismo ritmo que asciende el volumen de información (Montoya, Hernández, \& Ramos, 2016). Finalmente las ciencias de la informática y tratamiento de información, son 
disciplinas en las que intervienen múltiples habilidades y competencias para la vida, aplicables a otras áreas del conocimiento, aportando nuevas formas de razonar, solucionar dificultades y comprender el entorno. El territorio se ha convertido en aspecto simbólico y de afirmación de sus condiciones (Elía \& Elía, 2014).

Finalmente, es necesario ajustar constantemente el proceso de aprendizaje de acuerdo con el entorno y edad, para garantizar los mejores resultados para los futuros emprendedores y mantener los estándares e indicadores de desempeño. La actitud está altamente influenciada por las características socioeconómicas y culturales, que determinaron su comportamiento como consecuencia de las experiencias, formación, otros factores de su entorno, reconociendo su rol como actor activo de la misma. Las respuestas actitudinales favorables de su entorno familiar propiciaron la participación proactiva de los niños y jóvenes en las actividades de fortalecimiento de la competencia emprendedora.

\section{Reconocimiento}

Los investigadores agradecen a la Universidad Pedagógica y Tecnológica de Colombia (Proyectos SGI 2870 y 2684), Gobernación de Boyacá, Secretaría de Productividad, TIC y Gestión del Conocimiento por el apoyo financiero, junta de acción comunal y población de los Cojines del Zaque del Municipio de Tunja.

\section{Referencias}

Behar, D. (2008). Metodología de la Investigación (A. Rubeira). Editorial Shalom 2008.

Benavides, M. D. M., Sánchez, M. I., \& Luna, R. (2010). El proceso de aprendizaje para los emprendedores en la situacion actual: un analisis cualitativo en el ambito universitario. $D$ y $O, 30-48$. Retrieved from http://revistadyo.com/index.php/dyo/article/viewFile/122/122

Bonilla-del-río, M., Diego-Mantecón, J., \& Lena-Acebo, F. (2018). Estudiantes Universitarios : prosumidores de recursos digitales y mediáticos en la era de internet. Aula Abierta, 47(1972), 8. https://doi.org/https://doi.org/10.17811/rifie.47.3.2018.319-326

César García - Rincón de Castro. (2013). El emprendegrama: instrumento para evaluar la capacidad emprendedora del alumnado de ESO y Bachillerato. 1-8. Retrieved from https://s3.eu-west1.amazonaws.com/eu.storage.safecreative.org/1/2013/06/11/0000013f/3230/5076/65f8/d803e1e18ef4/ Emprendegrama.pdf?response-content-type=application\%2Fpdf\&X-Amz-Algorithm=AWS4-HMACSHA256\&X-Amz-Date=20201009T161408Z\&X-Amz-SignedHeaders=host\&X-Amz-Expires=86400\&X-AmzCredential=1SXTY4DXG6BJ3G4DXHR2\%2F20201009\%2Feu-west-1\%2Fs3\%2Faws4_request\&X-AmzSignature $=612 \mathrm{e} 87 \mathrm{~d} 49 \mathrm{~d} 147 f 6 a 8 c 64 d 24 a 3222145 \mathrm{~d} 0 \mathrm{c} 1 \mathrm{~d} 7 \mathrm{accbc3daf1e36fdd27d65423841}$

Cotoi, E., Bodoasca, T., Catana, L., \& Cotoi, I. (2011). Entrepreneurship European development strategy in the field of education. Procedia - Social and Behavioral Sciences, 15, 3490-3494.

https://doi.org/https://doi.org/10.1016/j.sbspro.2011.04.323

Cuervo, A. A. V., Vázquez, F. I. G., Acuña, G. M. T., Murrieta, M. U., \& Quiñonez, C. S. G. (2020). Medición en Investigación Educativa con Apoyo del SPSS y el AMOS Fernanda Inéz García Vázquez. Retrieved from https://www.researchgate.net/profile/Fernanda_Garcia_Vazquez/publication/341622791_Medicion_en_I nvestigacion_Educativa_con_Apoyo_del_SPSS_y_el_AMOS/links/5ef6118f92851c52d6fdf302/Medicionen-Investigacion-Educativa-con-Apoyo-del-SPSS-y-el-AMOS.pdf

Deste, P., Mahdi, S., Neely, A., \& Rentocchini, F. (2012). Inventors and entrepreneurs in academia: What types of skills and experience matter? Technovation, 32(5), 293-303.

https://doi.org/10.1016/j.technovation.2011.12.005 
Doğan, E. (2016). THE EFFECT OF ENTREPRENEURSHIP EDUCATION ON ENTREPRENEURIAL INTENTIONS OF UNIVERSITY STUDENTS IN TURKEY. Istanbul University Econometrics and Statistics E-Journal, (23), 79-93. Retrieved from https://dergipark.org.tr/tr/pub/iuekois/issue/27283/287198

Elía, V. de, \& Elía, P. de. (2014). Niños creadores de tecnología. 1-14.

Elia, G., Margherita, A., \& Passiante, G. (2020). Digital entrepreneurship ecosystem: How digital technologies and collective intelligence are reshaping the entrepreneurial process. Technological Forecasting and Social Change, 150, 119791. https://doi.org/https://doi.org/10.1016/j.techfore.2019.119791

Expertemprende. (2015). Guía de habilidades emprendedoras.

Fernández, M. (2014). La iniciativa emprendedora en edades tempranas (Marpadal Interactive Media S.L, Ed.). Retrieved from https://books.google.com.co/books/about/La_iniciativa_emprendedora_en_edades_tem.html?id=6z6FBQ AAQBAJ\&redir_esc=y

Fitzsimmons, T. W., \& Callan, V. J. (2020). The diversity gap in leadership: What are we missing in current theorizing? The Leadership Quarterly, 31(4), 101347. https://doi.org/https://doi.org/10.1016/j.leaqua.2019.101347

Gieure, C., Benavides-Espinosa, M. del M., \& Roig-Dobón, S. (2020). The entrepreneurial process: The link between intentions and behavior. Journal of Business Research, 112(January), 541-548. https://doi.org/10.1016/j.jbusres.2019.11.088

Gobernación de Boyacá. (2011). FASE II DEL PLAN ESPECIAL DE MANEJO Y PROTECCIÓN -PEMP-BIEN DE INTERES CULTURAL COJINES DEL ZAQUE, UBICADO EN LA CIUDAD DE TUNJA, DEPARTAMENTO DE BOYACA.

Guti, L. (2019). Cómo realizar e interpretar un análisis factorial exploratorio utilizando SPSS. REIRE Revista d'Innovació i Recerca En Educació, 12(2), 1-14. https://doi.org/10.1344

Haugh, H. M., \& Talwar, A. (2016). Linking Social Entrepreneurship and Social Change: The Mediating Role of Empowerment. Journal of Business Ethics, 133(4), 643-658. https://doi.org/10.1007/s10551-014-2449-4

Hernandez Sampieri, R., Fernandez Collado, C., \& Baptista Lucio, M. del P. (2010). Metodología de la investigación. In Metodología de la investigación. https://doi.org/- ISBN 978-92-75-32913-9

Huber, L. R., Sloof, R., \& Van Praag, M. (2014). The effect of early entrepreneurship education: Evidence from a field experiment. European Economic Review, 72, 76-97.

https://doi.org/https://doi.org/10.1016/j.euroecorev.2014.09.002

Ismail, V. Y., Zain, E., \& Zulihar. (2015). The Portrait of Entrepreneurial Competence on Student Entrepreneurs. Procedia - Social and Behavioral Sciences, 169, 178-188. https://doi.org/https://doi.org/10.1016/j.sbspro.2015.01.300

Jena, R. K. (2020). Measuring the impact of business management Student's attitude towards entrepreneurship education on entrepreneurial intention: A case study. Computers in Human Behavior, 107(January), 106275. https://doi.org/10.1016/j.chb.2020.106275

Kabukcu, E. (2015). Creativity Process in Innovation Oriented Entrepreneurship: The case of Vakko. Procedia Social and Behavioral Sciences, 195, 1321-1329.

https://doi.org/https://doi.org/10.1016/j.sbspro.2015.06.307 
Kantis, H. (2017). El emprendimiento juvenil: presente y posibilidades en América Latina. Prodem, Investigación, Acción, Aprendizaje.

Karlidag-Dennis, E., Hazenberg, R., \& Dinh, A.-T. (2020). Is education for all? The experiences of ethnic minority students and teachers in North-western Vietnam engaging with social entrepreneurship. International Journal of Educational Development, 77, 102224.

https://doi.org/https://doi.org/10.1016/j.ijedudev.2020.102224

Kimmitt, J., Muñoz, P., \& Newbery, R. (2020). Poverty and the varieties of entrepreneurship in the pursuit of prosperity. Journal of Business Venturing, 35(4), 105939.

https://doi.org/https://doi.org/10.1016/j.jbusvent.2019.05.003

La Guardia, D., Gentile, M., Dal Grande, V., Ottaviano, S., \& Allegra, M. (2014). A Game based Learning Model for Entrepreneurship Education. Procedia - Social and Behavioral Sciences, 141, 195-199. https://doi.org/https://doi.org/10.1016/j.sbspro.2014.05.034

López, K. S., \& González, G. T. (2014). Métodos y técnicas cualitativas y cuantitativas aplicables a la investigación en ciencias sociales (Tirant Hum). Retrieved from http://eprints.uanl.mx/13416/1/2014_LIBRO Metodos y tecnicas_Aplicacion del metodo pag499_515.pdf

Martínez, F., \& Carmona, G. (2009). Aproximación al Concepto de "Competencias Emprendedoras ": Valor Social e Implicaciones Educativas. REICE. Revista Iberoamericana Sobre Calidad, Eficacia y Cambio En Educación, 7(3), 5403.

Martinez, O. (2018). Uso de las Tecnologías de la Información y la Comunicación en la Educación Básica. Revista Scientific. https://doi.org/https://doi.org/10.29394/Scientific.issn.2542-2987.2018.3.10.8.154-174

Mateus, C., \& Galeano, P. (2016). Habilidades emprendedoras en población infantil. 3er Simposio Internacional de Investigación En Ciencias Económicas, Administrativas y Contables - Sociedad y Desarrollo, 23. Retrieved from http://www.unilibre.edu.co/bogota/pdfs/2016/3sin/B28.pdf

Montoya, A., Hernández, J., \& Ramos, H. (2016). Analizar por qué se debe enseñar la lógica de programación a niños y jóvenes en los hogares de la ciudad de Medellín . Revista CIES -, 7(10), 2-14.

Pico, J. (2012). Reseña de "Cómo formar niños y niñas con espíritu emprendedor. Manual para el formador." Revista Escuela de Administración de Negocios, (73), 241-242.

Portillo-Torres, M. (2017). Educación por habilidades : Perspectivas y retos para el sistema educativo. Revista Educación, 41(2), 13. https://doi.org/http://dx.doi.org/10.15517/revedu.v41i2.21719

Programa de las Naciones Unidas para el Desarrollo (PNUD). (2016). Promoción del emprendimiento y la innovación social juvenil en America Latina.

Rekalde, I., Vizcarra, M. T., \& Macazaga, A. M. (2013). La Observación Como Estrategia De Investigación Para Construir Contextos De Aprendizaje Y Fomentar Procesos Participativos. Educacion XX1, 17(1), 201-220. https://doi.org/10.5944/educxx1.17.1.1074

Rosendahl Huber, L., Sloof, R., Van Praag, M., \& Parker, S. C. (2020). Diverse cognitive skills and team performance: A field experiment based on an entrepreneurship education program. Journal of Economic Behavior \& Organization, 177, 569-588. https://doi.org/https://doi.org/10.1016/j.jebo.2020.06.030 
Ruiz, A. C., \& Uribe, J. V. (2013). Usos iniciales y desusos de la estrategia "Habilidades digitales para todos" en escuelas secundarias de Veracruz. Perfiles Educativos, 35(142), 8-26. https://doi.org/https://doi.org/10.1016/S0185-2698(13)71846-4

Sampieri, R. H. (2014). Metodologia de la investigación (McGRAW-HIL). México D.F.

Sánchez, J., Ward, A., Hernández, B., \& Florez, J. (2017). Educación emprendedora : Estado del arte. Propósitos y Representaciones, 5(2), 401-437.

Santos, G., Marques, C. S., \& Ferreira, J. J. M. (2020). Passion and perseverance as two new dimensions of an Individual Entrepreneurial Orientation scale. Journal of Business Research, 112(August 2019), 190-199. https://doi.org/10.1016/j.jbusres.2020.03.016

Sigalat Signes, E., Calvo Palomares, R., Roig Merino, B., \& Buitrago Mera, J. M. (2019). La investigación acción participativa (IAP) en el sector empresarial. interviniendo desde lo local. Empiria. Revista de Metodología de Ciencias Sociales, (44), 47-78. https://doi.org/10.5944/empiria.44.2019.25352

Tadeu, P., \& Paiva, T. (2015). Gamentship - An Innovative Project to Improve Entrepreneurship Competences. Procedia - Social and Behavioral Sciences, 174, 1829-1833.

https://doi.org/https://doi.org/10.1016/j.sbspro.2015.01.844

Toma, S.-G., Grigore, A.-M., \& Marinescu, P. (2014). Economic Development and Entrepreneurship. Procedia Economics and Finance, 8, 436-443. https://doi.org/https://doi.org/10.1016/S2212-5671(14)00111-7

Torimiro, D. O., \& Dionco-Adetayo, E. A. (2005). Children involvement in entrepreneurship in rural communities: an attitudinal analysis. Technovation, 25(6), 683-689.

https://doi.org/https://doi.org/10.1016/j.technovation.2003.09.011

Vilcov, N., \& Dimitrescu, M. (2015). Management of Entrepreneurship Education: A Challenge for a Performant Educational System in Romania. Procedia - Social and Behavioral Sciences, 203, 173-179.

https://doi.org/https://doi.org/10.1016/j.sbspro.2015.08.278

Esta obra está bajo una Licencia Creative Commons

Attribución-NoCommercial 4.0 International

(cc) BY-NC 